\title{
Clinical outcomes and aetiology of fourth cranial nerve palsy with acute vertical diplopia in adults
}

\author{
Shin Yeop $\mathrm{Oh}^{1} \cdot$ Sei Yeul $\mathrm{Oh}^{2}$
}

Received: 10 June 2019 / Revised: 2 December 2019 / Accepted: 9 December 2019 / Published online: 13 January 2020

(c) The Author(s), under exclusive licence to The Royal College of Ophthalmologists 2020

\begin{abstract}
Background We investigated the clinical outcomes of fourth cranial nerve (CN4) palsy with acute vertical diplopia in adults. Methods A total of 80 patients with acute CN4 palsy who underwent at least 3 months of follow-up were included in this study. We retrospectively investigated the aetiology, rate of recovery, and factors associated with recovery between March 2016 and January 2019.

Results The average age of patients with CN4 palsy was about 60 years, and the duration of recovery was 1.5 months: 48 $(60.0 \%)$ patients had a vascular aetiology and $17(21.3 \%)$ patients had a trauma history. Brain lesions were found in four (5.0\%) patients and decompensated cause accounted for four (5.0\%) cases. Among the total of 80 patients, 13 (16.3\%) failed to completely recover. Non-isolated CN4 palsy with other cranial nerve palsies were recorded in seven cases. The comparison between recovery and non-recovery groups showed that initial deviation angle, aetiology, fundus extorsion, and head tilt status were significantly different factors.

Conclusion The recovery rate of acute $\mathrm{CN} 4$ palsy was about $80 \%$ and duration of recovery was 1.5 months. However, the varying rates and duration of recovery was presented according to aetiology thus we should consider the prognosis by aetiology.
\end{abstract}

\section{Introduction}

Fourth cranial nerve (CN4) palsy is the most frequent cause of acquired vertical diplopia [1]. In fact, CN4 palsy constitutes a large proportion of patients who are treated for acute vertical diplopia in clinical practice. Although many studies investigated the causes of third, fourth, and sixth cranial nerve palsy [2-4], few analysed CN4 palsy only [5-7]. According to a previous report, decompensation of congenital CN4 palsy is the most frequent cause of acute vertical diplopia $[8,9]$. In a few patients diagnosed with congenital CN4 palsy, the range of fusion may be diminished by aging, stress or fatigue, especially in patients with a monocular decreased visual acuity [6]. However, vascular aetiology appears to be the

Sei Yeul Oh

mojili914@hanmail.net

1 Department of Ophthalmology, Samsung Changwon Hospital, Sungkyunkwan University School of Medicine, Changwon, Korea

2 Department of Ophthalmology, Samsung Medical Center, Sungkyunkwan University School of Medicine, Seoul, Korea main cause of acquired cranial nerve palsies, according to recent studies $[3,4,6,10]$. In other studies regardless of diplopia, the diverse aetiology of CN4 palsy including congenital types have been defined, and congenital aetiology was responsible for the high proportion of CN4 palsy $[2,4,5]$. On the other hand, few studies investigated CN4 palsy in adult patients with acute vertical diplopia. Therefore, we performed this retrospective study to analyse the clinical outcomes and aetiology of only CN4 palsy associated with acute vertical diplopia in adults.

\section{Methods}

This study was approved by the Institutional Review Board of Samsung Changwon Hospital (Changwon, Republic of Korea) and conducted according to the tenets of the Declaration of Helsinki. We retrospectively reviewed the medical records of adult patients who presented with acute vertical diplopia associated with CN4 palsy from March 2016 to January 2019 at the Samsung Changwon Hospital. Patients who did not confirm recovery with absence of follow-up duration at least 3 months were excluded. Case 
Table 1 Demographics of fourth cranial nerve palsy with acute vertical diplopia in adult.

\begin{tabular}{|c|c|}
\hline \multicolumn{2}{|l|}{ Parameters } \\
\hline Total patients $(n)$ & 80 \\
\hline Male:female $(n)$ & $60(75.0 \%): 20(25.0 \%)$ \\
\hline Age at time of onset (years) & $59.96 \pm 11.87$ (range: $30-82$ ) \\
\hline Duration of follow-up (days) & $140.83 \pm 45.00$ \\
\hline Duration of recovery (days) & $45.65 \pm 35.14$ \\
\hline \multicolumn{2}{|l|}{ Recovery state $(n)$} \\
\hline Complete:none:partial & $\begin{array}{l}63(78.75 \%): 13 \\
(16.25 \%): 4(5.0 \%)\end{array}$ \\
\hline Involved eye (right:left) & $42(52.5 \%): 38(47.5 \%)$ \\
\hline $\begin{array}{l}\text { Deviation angle at primary } \\
\text { position (PD) }\end{array}$ & $4.16 \pm 2.99$ \\
\hline $\begin{array}{l}\text { Deviation angle at ipsilateral tilt } \\
\text { position }(\mathrm{PD})\end{array}$ & $7.19 \pm 4.22$ \\
\hline \multicolumn{2}{|l|}{ Aetiology } \\
\hline Vascular & $48(60.0 \%)$ \\
\hline Trauma & $17(21.25 \%)$ \\
\hline Brain lesion & $4(5.0 \%)$ \\
\hline Late decompensation & $4(5.0 \%)$ \\
\hline Others & $4(5.0 \%)$ \\
\hline Undetermined & $3(3.75 \%)$ \\
\hline Presence of fundus extorsion $(n)$ & $49(61.25 \%)$ \\
\hline Presence of head tilt $(n)$ & $24(30.0 \%)$ \\
\hline Other neurological symptoms $(n)$ & $12(15.0 \%)$ \\
\hline Hypertension $(n)$ & $23(28.75 \%)$ \\
\hline Diabetes mellitus $(n)$ & $19(23.75 \%)$ \\
\hline Dyslipidemia $(n)$ & $14(17.5 \%)$ \\
\hline Coronary vascular disease $(n)$ & $5(6.25 \%)$ \\
\hline Other cranial nerve palsy $(n)$ & $7(8.75 \%)$ \\
\hline Smoking $(n)$ & $22(27.5 \%)$ \\
\hline Brain MRI evaluation $(n)$ & $64(80.0 \%)$ \\
\hline
\end{tabular}

Values are presented as mean $\pm \mathrm{SD}$

$S D$ standard deviation, $P D$ prism dioptres; $M R I$ magnetic resonance imaging

notes were reviewed recording demographics, aetiology, neuroimaging, interval to symptom-resolution, and recovery. The aetiology recorded in this study was established by the attending neuro-ophthalmologist. Neuroimaging was not a routine examination and was at the discretion of the neuro-ophthalmologist. The criteria for CN4 palsy were hypertropia in straight-ahead gaze, increasing with opposite lateral gaze and same head tilt, underaction of the superior oblique muscle and/or overaction of its antagonistic inferior oblique muscle, and excyclotorsion as measured with the double Maddox rod test. Consecutive patients who had been followed up for $>3$ months were included in this study and patients with previously diagnosed paralytic or restrictive strabismus or previous extraocular muscle surgery were excluded. The patients' age of onset, sex, presence of head tilt, findings from brain magnetic resonance imaging (MRI), and previous medical history, especially the presence of vascular risk factors (hypertension, diabetes mellitus, dyslipidemia, and coronary vascular disease) were reviewed together with other neurological symptoms and smoking status. At first visit, patients underwent ophthalmologic assessment, including slit-lamp, measurement of vertical deviation, and fundus photography. The angle of deviation was measured using the alternating prism cover test in a primary position and both head tilt position at distant fixation. The fundus imaging was performed in all patients with upright primary gaze. A single neuro-ophthalmologist (ShYO) decided whether or not to have a fundus extorsion.

The patients were classified into the following six groups according to aetiology: vascular, trauma, brain lesion, late decompensation, others, and undetermined. The vascular group was defined as having at least one vascular risk factor (hypertension, diabetes mellitus, dyslipidemia, and coronary vascular disease) without a history of trauma or evidence of brain lesions in imaging studies. The trauma group was defined by a positive history of head trauma secondary to falls, traffic accidents, violence, or iatrogenic causes. The CN4 palsy caused by brain lesions was attributed to anatomically directly related cerebral haemorrhage, cerebral infarction, or brain tumour. Late decompensation was defined by patients without vascular risk factors or trauma, age younger than 40 years or unilateral visual loss. In these patients, the aetiology was established as decompensation after a detailed investigation regarding head tilt supported by old photo and the onset of diplopia, if present. Other causes included complications of other anatomical lesion and inflammation or systemic disease related to the cranial nerve function. Any idiopathic CN4 palsy with none of the above characteristics was categorised as undetermined. Based on presentation of symptom, only CN4 palsy was defined isolated group and $\mathrm{CN} 4$ palsy with other cranial nerve palsies was defined non-isolated group.

The prognosis at the follow-up final visit was deemed complete, partial, or non-recovery based on the deviation angle and ocular motor limitation. Complete recovery was defined as an absence of vertical deviation or ocular motor limitation, and partial recovery was defined as a decrease by more than $50 \%$ of the initial deviation angle. Nonrecovery was defined as the absence of complete and partial recovery or cases that underwent extraocular muscle surgery for recovery.

Statistical analyses were conducted by an independent statistician using a commercially available statistical package (SPSS V.18.0 for windows; SPSS, Chicago, IL, USA). Demographic differences between the groups were compared using Student's $t$ test, Fisher's exact test, and Pearson's chi-square test. A $p$ value less than 0.05 was considered statistically significant. 


\section{Results}

\section{Baseline characteristics and clinical outcomes according to aetiology}

A total of 80 patients (60 males and 20 females) were included in this study, and the mean length of follow-up was $140.83 \pm 45.0$ days after initial visit. The average age at CN4 palsy onset was $59.96 \pm 11.87$ years (range: $30-82$ ). The aetiology was classified into vascular $(60.0 \%)$, trauma (21.25\%), brain lesion (5.0\%), late decompensation (5.0\%), other $(5.0 \%)$, and undetermined $(3.75 \%)$ categories. Complete recovery was seen in 63 patients $(78.75 \%)$ and the mean duration of recovery was $45.65 \pm 35.14$ days. Partial recovery was observed in 4 patients $(5.0 \%)$ and nonrecovery was seen in 13 patients $(16.25 \%)$. Among the 80 patients, 49 (61.25\%) showed fundus extorsion, 24 (30.0\%) had a head tilt, 23 (28.75\%) exhibited hypertension, and 19 (23.75\%) manifested diabetes mellitus. Brain MRI was performed in 64 patients $(80.0 \%)$ (Table 1).

Among four patients with brain lesion, two had a brain stem infarction and one had meningioma. One patient with brain haemorrhage had an accompanying partial third nerve palsy due to red nucleus haemorrhage. Of these, three patients except brain tumour were completely recovered. Patients with other causes of CN4 palsy included two cases of Tolosa-Hunt syndrome, and one case each of ocular myasthenia gravis and fronto-ethmoidal mucocele. Tolosa-hunt syndrome was diagnosed by enhanced inflammation lesion at cavernous sinus in brain MRI and ipsilateral headache or periorbital pain. One patient of Tolosa-Hunt syndrome had an accompanied facial nerve palsy. Among four patients of late decompensation, two had a low vision due to monocular epi-retinal membrane and cataract. The complete recovery rate was $91.67 \%$ in patients with vascular causes, $64.7 \%$ in case of traumatic injuries, $75.0 \%$ involving brain lesions, and $75 \%$ in others. However, there was no complete recovery in patients with late decompensation.

\section{Comparison between groups with presentation (isolated vs non-isolated) and aetiology (vascular vs trauma)}

Patients were divided into two groups: isolated and nonisolated. The isolated CN4 palsy without other cranial nerve palsy are 73 patients and non-isolated $\mathrm{CN} 4$ palsy were recorded in seven cases. In non-isolated CN4 palsy, three had an ipsilateral facial nerve palsy, two had an ipsilateral sixth nerve palsy, one had an ipsilateral facial and sixth nerve palsy, and one patient had a partial third nerve palsy. Of these, three were patients of trauma aetiology, two patients had a brain lesion, one patient is vascular aetiology,
Table 2 Comparison characteristics between isolated and nonisolated group.

\begin{tabular}{lllr}
\hline Parameters & $\begin{array}{l}\text { Isolated } \\
(n=73)\end{array}$ & $\begin{array}{l}\text { Non-isolated } \\
(n=7)\end{array}$ & $p$ value \\
\hline Age at time of onset (years) & $60.67 \pm 11.65$ & $52.57 \pm 12.57$ & 0.087 \\
Male:female $(n)$ & $55: 18$ & $5: 2$ & $>0.999$ \\
Duration of follow-up (days) & $136.86 \pm 42.82$ & $182.14 \pm 49.73$ & 0.025 \\
Duration of recovery (days) & $48.59 \pm 38.09$ & $57.83 \pm 38.30$ & 0.351 \\
Aetiology $(n)$ & & & 0.014 \\
$\quad$ Vascular & $47(64.38 \%)$ & $1(14.29 \%)$ & \\
$\quad$ Trauma & $14(19.18 \%)$ & $3(42.86 \%)$ & \\
$\quad$ Brain lesion & $2(2.74 \%)$ & $2(28.57 \%)$ & \\
Late decompensation & $4(5.48 \%)$ & $0(0 \%)$ & \\
$\quad$ Others & $3(4.11 \%)$ & $1(14.29 \%)$ & \\
$\quad$ Undetermined & $3(4.11 \%)$ & $0(0 \%)$ & \\
Deviation angle at primary & $3.78 \pm 2.12$ & $8.14 \pm 6.59$ & 0.014 \\
position (PD) & & & \\
Deviation angle at ipsilateral tilt & $6.67 \pm 3.18$ & $12.57 \pm 8.75$ & 0.035 \\
position (PD) & & & \\
Presence of fundus extorsion $(n)$ & $43(58.91 \%)$ & $6(85.71 \%)$ & 0.132 \\
Presence of head tilt $(n)$ & $22(30.1 \%)$ & $2(28.57 \%)$ & $>0.999$ \\
Other neurological symptoms $(n)$ & $9(12.33 \%)$ & $3(42.86 \%)$ & 0.065 \\
Hypertension $(n)$ & $22(30.14 \%)$ & $1(14.29 \%)$ & 0.667 \\
Diabetes mellitus $(n)$ & $17(23.29 \%)$ & $2(28.57 \%)$ & 0.668 \\
Dyslipidemia $(n)$ & $13(17.81 \%)$ & $1(14.29 \%)$ & $>0.999$ \\
Coronary vascular disease $(n)$ & $5(6.85 \%)$ & $0(0 \%)$ & $>0.999$ \\
Smoking $(n)$ & $18(24.66 \%)$ & $4(57.14 \%)$ & 0.086 \\
Brain MRI evaluation $(n)$ & $58(79.45 \%)$ & $6(85.71 \%)$ & $>0.999$ \\
\hline Values & & & \\
\hline
\end{tabular}

Values are presented as mean \pm SD

$S D$ standard deviation, $P D$ prism dioptres, $M R I$ magnetic resonance imaging

and one patient was diagnosed Tolosa-hunt syndrome. Two patients with brain lesion are brain stem infarction and red nucleus haemorrhage. In non-isolated group, one traumatic CN4 palsy was not recovered and required surgery and other six patients were completely recovered. In two patients of isolated group with structural brain lesion, one patient had a meningioma and one patient had a pons infarction. The aetiology showed significant difference (isolated: vascular 64.38\%; non-isolated: trauma and brain lesion $71.43 \%, p<0.014)$. And the initial deviation angle at primary and tilt position were significantly smaller in the isolated group than in the non-isolated groups $(p=0.014$, $p=0.035)$ (Table 2).

We compared the two groups of vascular and traumatic cases accounting for almost $80 \%$ of the aetiologies. Age at the time of onset $(p=0.001)$, recovery rate $(p=0.008)$, and hypertension $(p=0.006)$ were significantly different factors (Table 3).

\section{Comparison between groups with complete recovery vs partial recovery and non-recovery}

Patients were divided into two groups: complete recovery, partial, and non-recovery. The aetiology showed significant difference (complete: vascular $69.84 \%$, trauma $17.46 \%$; 
Table 3 Comparison characteristics between vascular and trauma group.

\begin{tabular}{lllr}
\hline Parameters & Vascular $(n=48)$ & Trauma $(n=17)$ & $p$ value \\
\hline Age at time of onset (years) & $63.77 \pm 9.60$ & $53.29 \pm 11.33$ & 0.001 \\
Male:female $(n)$ & $36: 12$ & $13: 4$ & $>0.999$ \\
Duration of follow-up (days) & $134.31 \pm 35.39$ & $128.71 \pm 46.84$ & 0.516 \\
Duration of recovery (days) & $49.40 \pm 35.17$ & $39.55 \pm 39.13$ & 0.188 \\
Recovery state $(n)$ & & & \\
$\quad$ Complete:none + partial & $44(91.67 \%): 4$ & $11(64.7 \%): 6$ & 0.008 \\
Deviation angle at primary position (PD) & $3.67 \pm 1.65$ & $3.65 \pm 3.12$ & 0.328 \\
Deviation angle at ipsilateral tilt position $(\mathrm{PD})$ & $6.69 \pm 2.71$ & $6.53 \pm 5.15$ & 0.223 \\
Presence of fundus torsion $(n)$ & $27(56.25 \%)$ & $8(47.06 \%)$ & 0.438 \\
Presence of head tilt $(n)$ & $10(20.83 \%)$ & $5(9.41 \%)$ & 0.512 \\
Other neurological symptoms $(n)$ & $7(14.58 \%)$ & $0(0 \%)$ & 0.176 \\
Hypertension $(n)$ & $21(43.75 \%)$ & $1(5.88 \%)$ & 0.006 \\
Diabetes mellitus $(n)$ & $15(31.25 \%)$ & $4(23.53 \%)$ & 0.758 \\
Dyslipidemia $(n)$ & $12(25.0 \%)$ & $2(11.76 \%)$ & 0.254 \\
Coronary vascular disease $(n)$ & $5(10.42 \%)$ & $0(0 \%)$ & 0.315 \\
Other cranial nerve palsy $(n)$ & $1(2.08 \%)$ & $3(17.65 \%)$ & 0.052 \\
Smoking $(n)$ & $14(29.17 \%)$ & $4(23.53 \%)$ & 0.655 \\
Brain MRI evaluation $(n)$ & $40(83.33 \%)$ & $14(82.35 \%)$ & 0.926 \\
\hline Vales & & &
\end{tabular}

Values are presented as mean $\pm \mathrm{SD}$

$S D$ standard deviation, $P D$ prism dioptres, $M R I$ magnetic resonance imaging partial and non-recovery: vascular $23.53 \%$, trauma $35.29 \%$, $p<0.001)$. In four patients showing partial recovery, the recovery period was about 4 months $(108.75 \pm 33.13$ days $)$ compared with 1.5 months for complete recovery $(p=$ 0.006). Further, the initial deviation angle at primary and tilt position were significantly smaller in the complete recovery group than in the partial and non-recovery groups $(p=$ $0.036, p=0.041)$. Fundus extorsion and head tilt were significantly different factors $(p=0.029, \quad p<0.001)$ (Table 4).

\section{Discussion}

Acute cranial nerve palsy may be an early sign of serious intracranial lesion, warranting careful assessment including neuroimaging. However, benign aetiologies such as microvascular disease and decompensation are by far the most common [2, 5]. Previous studies reported recovery rates of $38-85 \%$ for the cranial nerve palsy $[2-4,10]$. The wide range of recovery rates is attributed to variable evaluation criteria and varying distribution of cranial nerve palsies in the studies. In most studies, CN4 palsy is rare and the frequency of sixth cranial nerve palsy is the most common. On the other hand, CN4 may be the cranial nerve most affected pathologically, whether directly or indirectly, due to its thin structure and long course along the tentorial edge [11]. Thus, they are thought to be less vulnerable to the mass effect and therefore, CN4 palsy is known to occur rarely by tumour and aneurysm [2, 3]. In the group of patients with brain lesions in our study, only a single patient had a brain tumour and none had an aneurysm. Our data confirmed the benign aetiology of most acute $\mathrm{CN} 4$ palsies, which resolved spontaneously as reported in other studies [4-6]. However, because there were two brain lesions in the isolated group, it should be prudent to exclude brain lesions in all CN4 palsy. The most common aetiology for acute CN4 palsy, based on our classification, were vascular and trauma. Park et al. [4] reported that vascular and traumatic causes were the predominant aetiology of acquired CN4 palsy as reported in our study. By contrast, previous studies $[5,6]$ reported that congenital or microvascular factors were the most frequent cause. These results can be explained by the different referral patterns based on the population distribution. It is also relevant to analyse only CN4 palsy patients with acute vertical diplopia. On the other hand, in seven non-isolated CN4 palsy, only one patient is a vascular cause, three are trauma aetiology, and two had a brain lesion. Therefore, if non-isolated CN4 palsy, we should consider other aetiology before vascular. The complete recovery rate was about $80 \%$ and the duration of recovery ranged from 2 weeks to 3 months in our study suggesting that most $\mathrm{CN} 4$ palsies showed complete recovery within 3 months.

Compared with the two predominant causes (vascular and trauma), age at time of onset, recovery rate, and hypertension showed statistically significant difference. The higher incidence of traumatic CN4 palsy at young ages may 
Table 4 Comparison characteristics between complete recovery and partial or nonrecovery group.

\begin{tabular}{|c|c|c|c|}
\hline Parameters & $\begin{array}{l}\text { Complete recovery } \\
(n=63)\end{array}$ & $\begin{array}{l}\text { Partial + non-recovery } \\
(n=17)\end{array}$ & $p$ value \\
\hline Age at time of onset (years) & $60.57 \pm 11.49$ & $57.71 \pm 13.30$ & 0.455 \\
\hline Male:female $(n)$ & $48: 15$ & $12: 5$ & 0.753 \\
\hline Duration of follow-up (days) & $139.84 \pm 43.32$ & $144.47 \pm 52.06$ & 0.715 \\
\hline Duration of recovery (days) & $45.65 \pm 35.14$ & $108.75 \pm 33.13$ & 0.006 \\
\hline Aetiology $(n)$ & & & $<0.001$ \\
\hline Vascular & $44(69.84 \%)$ & $4(23.53 \%)$ & \\
\hline Trauma & $11(17.46 \%)$ & $6(35.29 \%)$ & \\
\hline Brain lesion & $3(4.76 \%)$ & $1(5.88 \%)$ & \\
\hline Late decompensation & $0(0 \%)$ & $4(23.53 \%)$ & \\
\hline Others & $3(4.76 \%)$ & $1(5.88 \%)$ & \\
\hline Undetermined & $2(3.17 \%)$ & $1(5.88 \%)$ & \\
\hline Deviation angle at primary position (PD) & $3.94 \pm 2.96$ & $5.0 \pm 3.04$ & 0.036 \\
\hline $\begin{array}{l}\text { Deviation angle at ipsilateral tilt } \\
\text { position (PD) }\end{array}$ & $6.83 \pm 4.01$ & $8.53 \pm 4.82$ & 0.041 \\
\hline Presence of fundus extorsion $(n)$ & $34(53.97 \%)$ & $15(88.23 \%)$ & 0.029 \\
\hline Presence of head tilt $(n)$ & $11(17.46 \%)$ & $13(76.47 \%)$ & $<0.001$ \\
\hline Other neurological symptoms $(n)$ & $10(15.87 \%)$ & $2(11.76 \%)$ & 0.674 \\
\hline Hypertension $(n)$ & $20(31.75 \%)$ & $3(17.65 \%)$ & 0.368 \\
\hline Diabetes mellitus $(n)$ & $15(23.81 \%)$ & $4(23.53 \%)$ & 0.981 \\
\hline Dyslipidemia $(n)$ & $11(17.46 \%)$ & $3(17.65 \%)$ & 0.986 \\
\hline Coronary vascular disease $(n)$ & $3(4.76 \%)$ & $2(11.76 \%)$ & 0.290 \\
\hline Other cranial nerve palsy $(n)$ & $6(9.52 \%)$ & $1(5.88 \%)$ & 0.637 \\
\hline Smoking $(n)$ & $18(28.57 \%)$ & $4(23.53 \%)$ & 0.679 \\
\hline Brain MRI evaluation $(n)$ & $53(84.13 \%)$ & $11(64.71 \%)$ & 0.076 \\
\hline
\end{tabular}

Values are presented as mean $\pm \mathrm{SD}$

$S D$ standard deviation, $P D$ prism dioptres, $M R I$ magnetic resonance imaging be attributed to high risk of trauma due to increased physical activity. Vascular disease was the most common cause of acute CN4 palsy in our study. The rate of complete recovery in cases involving vascular aetiology was $91.7 \%$ except for four patients. These results are similar in that 93.5\% of presumed microvascular $\mathrm{CN} 4$ palsies resolved within 2 to 3 months in other study [5]. Also, $43.8 \%$ of patients of vascular aetiology were associated with hypertension compared with about 50\% in another study [5].

We classified prognosis into complete, partial, and nonrecovery. The group with complete recovery was compared with partial and non-recovery groups. The time to partial recovery was about 4 months, which was longer than 1.5 months in the complete group. This means that even if there is no improvement within $2-3$ months, the disease should be observed for more than 4 months. The aetiology, initial deviation angle, fundus extorsion, and head tilt were significantly different in patients with and without complete recovery. Vascular aetiology was the most common cause in patients showing complete recovery whereas trauma, brain lesion, and late decompensation accounted for $76 \%$ of all cases in partial and non-recovery group. In previous studies [4-6] reported that differences in aetiology in the recovered group were attributed to variable and ill-defined criteria. This study only investigated CN4 palsy, and therefore, was difficult to directly compare the recovery rate and aetiological factors with previous reports of cranial nerve palsy. However, the difference in the aetiology of CN4 palsy according to the recovery status can be used as a basis to predict the prognosis.

Initial deviation angle at primary or ipsilateral tilt positions were smaller in the complete recovery group similar to other studies [4]. This result can be explained by the fact that the angle of deviation at vascular is smaller than other causes of trauma or brain lesion and because vascular cause is the most common in complete recovery group [4]. However, the differences of 1-2 prism dioptres was not clinical significantly different. Further, head tilt was more common in the partial and non-recovery groups, and most of the patients with distinctive head tilt sustained trauma, brain lesion, and late decompensation. The factors contributing to delayed presentation of CN4 palsy are unclear, however, it is likely that the control of the deviation decompensates over time, possibly exacerbated by factors 
such as the onset of presbyopia and the use of downgaze for reading when wearing a presbyopic spectacle correction [12]. Other study explained that decompensation occurs to deteriorating fusion ability with long periods of monocular deprivation [6]. In the late decompensation group in this study, one patient exhibited a monocular dense cataract and another patient showed a monocular thick epi-retinal membrane. On the other hand, two patients with Tolosa-Hunt syndrome on the group showed complete recovery, suggesting a favourable prognosis for $\mathrm{CN} 4$ palsy induced by inflammation.

Our study had several limitations. First, it was a retrospective single-hospital-based study, suggesting a possible selection bias, and the results may differ according to the population or geographic location. Second, despite evaluation of the entire medical records, it is possible that aetiology or the underlying disease was incorrectly assigned in a few cases partly because of errors in medical history. Third, the differential diagnosis on vascular and undetermined aetiologies may be inaccurate due to base on the presence of vascular risk factors. Nevertheless, this study has an advantage of studying only CN4 palsy with acute vertical diplopia compared with previous studies analysing the entire third, fourth, and sixth cranial nerve palsies. In conclusion, our study demonstrated that vascular aetiology was the most common cause of acute $\mathrm{CN} 4$ palsy, and trauma ranked second among the possible causative factors. The overall rate of complete recovery from acute $\mathrm{CN} 4$ palsy was $78.8 \%$, however, because the prognosis and natural course varied depending on aetiology, we should consider a prognosis based on aetiology.

\section{Summary}

\section{What was known before}

- The fourth cranial nerve palsy is the most frequent cause of acquired vertical diplopia.

- Although many studies investigated the causes of third, fourth, and sixth cranial nerve palsy, few analysed fourth cranial nerve palsy only.

- Various aetiology such as congenital, microvascular disease, or trauma were reported.

\section{What this study adds}

- We performed this retrospective study to analyse the clinical outcomes and aetiology of only fourth cranial nerve palsy associated with acute vertical diplopia in 80 adults.

- The overall rate of complete recovery from acute fourth cranial nerve palsy was $78.8 \%$ and most common aetiology was vascular cause.

- The prognosis and natural course varied depending on aetiology.

Author contributions The conception or design of the work (ShYO, $\mathrm{SeYO}$ ); analysis and interpretation (ShYO); data collection and drafting the work (ShYO); critical revision of the article (SeYO); final approval of the version (SeYO).

\section{Compliance with ethical standards}

Conflict of interest The authors declare that they have no conflict of interest.

Publisher's note Springer Nature remains neutral with regard to jurisdictional claims in published maps and institutional affiliations.

\section{References}

1. Bielschowsky A. Lectures on motor anomalies of the eyes: II. Paralysis of individual eye muscles. Arch Ophthalmol. 1935; 13:33-59.

2. Richards BW, Jones FR, Younge BR. Causes and prognosis in 4278 cases of paralysis of the oculomotor, trochlear, and abducens cranial nerves. Am J Ophthalmol. 1992;113:489-96.

3. Tiffin PA, MacEwen CJ, Craig EA, Clayton G. Acquired palsy of the oculomotor, trochlear and abducens nerves. Eye. 1996;10: 377-84.

4. Park UC, Kim SJ, Hwang JM, Yu YS. Clinical features and natural history of acquired third, fourth, and sixth cranial nerve palsy. Eye. 2008;22:691-6.

5. Mollan SP, Edwards JH, Price A, Abbott J, Burdon MA. Aetiology and outcomes of adult superior oblique palsies: a modern series. Eye. 2009;23:640-4.

6. Khaier A, Dawson E, Lee J. Clinical course and characteristics of acute presentation of fourth nerve paresis. J Pediatr Ophthalmol Strabismus. 2012;49:366-9.

7. Dosunmu EO, Hatt SR, Leske DA, Hodge DO, Holmes JM. Incidence and etiology of presumed fourth cranial nerve palsy: a population-based study. Am J Ophthalmol. 2018;185:110-4.

8. Von Noorden GK, Murray E, Wong S. Superior oblique paralysis, a review of 270 cases. Arch Ophthalmol. 1986;104:1771-6.

9. Keane JR. Fourth nerve palsy: historical review and study of 215 in patients. Neurology. 1993;43:2439-43.

10. Berlit P. Isolated and combined pareses of cranial nerves III, IV, and VI. A retrospective study of 412 patients. J Neurol Sci. 1991;103:10-15.

11. Morris RJ. Double vision as a presenting symptom in an ophthalmic casualty department. Eye. 1991;5:124-9.

12. Plager DA. Superior oblique palsy and superior oblique myokymia. In: Rosenbaum AL, Santiago AP, editors. Clinical strabismus management principles and surgical techniques. Philadelphia: W. B. Saunders Company; 1999. 219-29. 